\title{
Molecular Dipole Osmosis Based on Induced Charge Electro-Osmosis
}

\author{
Hideyuki Sugioka* \\ Department of Mechanical Systems Engineering, \\ Shinshu University 4-17-1 Wakasato, Nagano 380-8553, Japan
}

(Dated: July 28, 2016)

\begin{abstract}
We propose a novel mechanism of producing a large nonlinear electrokinetic vortex flow around a nonconductive polar molecule in an electrolyte. That is, a large nonlinear electrokinetic slip velocity is derived by considering a local giant permittivity due to a molecular electric dipole moment with induced-charge electro-osmosis (ICEO). Different from the conventional ICEO theory, our theory predicts that a nonconductive biomaterial, such as a base of a deoxyribonucleic acid (DNA) molecule, has a significantly high ICEO flow velocity because of its large local permittivity. We consider that our findings will contribute markedly to promising biomedical applications.
\end{abstract}

\footnotetext{
*E-mail: hsugioka@shinshu-u.ac.jp
} 


\section{INTRODUCTION}

Induced-charge electro-osmosis (ICEO), which is an electro-osmotic phenomenon caused by the interaction between a tangential electric field and an electric double layer resulting from the induced charge around a conductive material in an electrolyte, has attracted considerable attention because of the possibility of a wide range of biomedical applications along with the essential new physical insight into a surface science [1-3]. However, in general, there is no reason to believe that a biomaterial, such as a base of a deoxyribonucleic acid (DNA) molecule, would behave the same way as a conductive material. This is because the ICEO flow velocities around a dielectric material are generally low and negligible in a standard microfluidic geometry. Thus, the direct applications of ICEO to promising sensor devices are limited. Nevertheless, in the range of a molecular scale concerning a nanopore size (typically, 2-60 $\mathrm{nm}$ ) used in promising next-generation DNA sequencers [4], we can expect surprisingly high local ICEO flow velocities around the bases of a DNA molecule owing to their giant local permittivity due to the large molecular dipole moments in a confined space. As a result, we can expect that the ICEO flow around a base dominates the interaction between the base and a wall during the translocation of the DNA molecule through the nanopore, since we already showed that the ICEO flow around a polarizable particle contributes to both the attitude control of the particle [5] and the suppression of Brownian noise [6] because of the hydrodynamic interaction due to ICEO. Thus, the ICEO flow around a biomolecule is important and it can directly contribute to the development of innovative next-generation DNA sequencers, although the nanopore problem is beyond the scope of this study because of its high complexity. Therefore, in this study, we focus on describing a novel nonlinear electrokinetic slip velocity around a nonconductive polar molecule.

\section{THEORY}

\section{A. Basic concept of molecular dipole osmosis based on ICEO}

Figure 1 shows the basic concept of molecular dipole osmosis based on ICEO. As shown in Fig. 1, we consider an electric osmotic flow around a polar molecule [e.g., thymine (T)]. Here, the polar molecule has a large molecular dipole moment $P_{B}$ in a confined space (e.g., $P_{B}=4.72 \mathrm{D}$ and $r_{B}^{T}=0.330 \mathrm{~nm}$ for thymine [4]); thus, the local permittivity and ICEO 


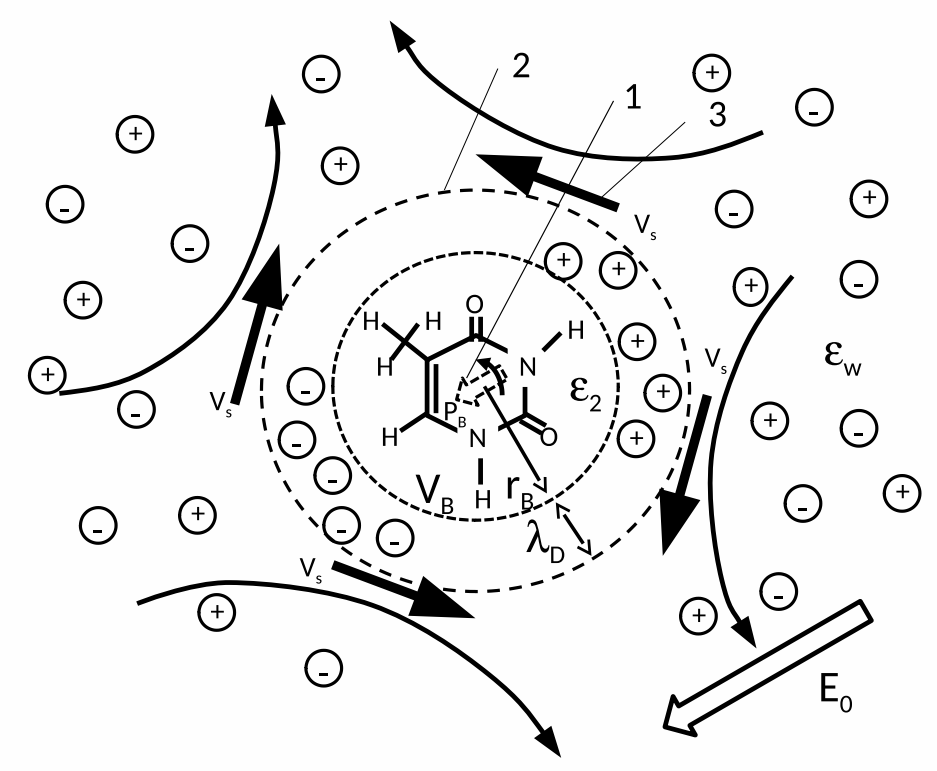

FIG. 1. Schematic view of a molecular dipole osmosis based on ICEO. 1: electric dipole moment. 2: electric double layer. 3: ICEO slip velocity. Here, we consider a large electric osmotic flow around a polar molecule [e.g., thymine $(\mathrm{T})]$ owing to a local giant permittivity (e.g., $\epsilon_{2}^{T}=46.1 \epsilon_{0}$ ) due to a large molecular dipole moment $P_{B}$ in a confined space (e.g., $P_{B}=4.72 \mathrm{D}$ and $r_{B}^{T}=0.330$ nm for thymine [4]).

flow velocity become high (e.g., $\epsilon_{2}^{T}=16.0 \epsilon_{0}$ and $U_{0, \epsilon_{2}, B}^{E_{0}=2 \mathrm{MV} / m}=0.802 \mathrm{~mm} / \mathrm{s}$ for thymine at $\lambda_{D}=10 \mathrm{~nm}$ as will be explained later). Namely, on the one hand, in the absence of an external electric field $E_{0}$, a polar molecule having the electric dipole moment $P_{B}$ rotates freely and thus the expectation value $\left\langle P_{B}>\right.$ for the single molecule becomes zero. On the other hand, by applying $E_{0}$, the probability that the direction of the dipole moment is parallel to the electric field increases and thus a nonzero value of $\left\langle P_{B}\right\rangle$ appears for a single molecule. Consequently, the molecular region of radius $r_{B}$ is polarized and the probabilistically induced charge attracts counter charge ions stochastically; then, two kinds of electric double layers are formed near the poles of the assumed polarizable particle of radius $r_{B}$. Furthermore, by the interaction between the tangential electric field and the ions in the electric double layer, a so-called slip velocity $V_{s}$ appears at the outside edge of the electric double layer of thickness $\lambda_{D}$; thus, a quadratic vortex flow appears stochastically around the polar molecule. 


\section{B. Zhao and Bau's ICEO formulations for a dielectric particle}

Zhao and Bau [7] already successfully formulated an ICEO flow around a dielectric particle having a thick electric double layer of thickness $\lambda_{D}$; i.e., when a $2 \mathrm{D}$ dielectric particle of relative permittivity $\epsilon_{r 2, B}$ is embedded in an infinite medium of relative permittivity $\epsilon_{r 1, w}$ and viscosity $\mu$ (typically, $1 \mathrm{mPa}$ s for water), the tangential flow velocity in the double layer is described as

$$
u^{2 D, \text { inside }}=2 U_{0, \epsilon_{r, B}}\left(1-\mathrm{e}^{-\frac{r-r_{B}}{\lambda_{D}}}\right) \sin 2 \theta
$$

where

$$
\begin{aligned}
& U_{0, \epsilon_{r 2, B}}=\chi U_{0}^{\infty}, \\
& \chi=\frac{1}{1+\frac{\epsilon_{r 1, w} r_{B}}{\epsilon_{r 2, B} \lambda_{D}}}, \\
& U_{0}^{\infty}=\frac{\epsilon_{w} r_{B} E_{0}^{2}}{\mu} .
\end{aligned}
$$

Note that $U_{0}^{\infty}$ denotes the ordinary representative slip velocity of an ICEO flow around the conductive particle of $\epsilon_{r 2, B}=\infty, U_{0, \epsilon_{r 2, B}}\left(=\chi U_{0}^{\infty}\right)$ denotes the representative slip velocity around the dielectric particle of $\epsilon_{r 2, B} \neq \infty$, and $\chi$ provides the ratio of the ordinary ICEO flow velocity of the conductive particle to the ICEO flow velocity of the dielectric particle. From Eq. (2), we can calculate the ICEO flow velocity around the dielectric particle by simply calculating $\chi$ and $U_{0}^{\infty}$. Furthermore, for typical microfluidic geometries, we can assume that $\epsilon_{r 2, B} \simeq 2$ to $10, \epsilon_{r 1, w} \simeq 80, \lambda_{D} \simeq 1$ to $100 \mathrm{~nm}$, and $r_{B} \simeq 1$ to $10 \mu \mathrm{m}$. Thus, typically $\epsilon_{r 1, w} / \epsilon_{r 2, B}=8$ to 40 and $r_{B} / \lambda_{D}=10$ to $10^{4}$; i.e., $\epsilon_{r 1, w} r_{B} / \epsilon_{r 2, B} \lambda_{D}=80$ to $8 \times 10^{5}$ and $\chi \simeq 10^{-2}$ to $10^{-6}$. Therefore, in general, the ICEO flow velocity around a dielectric particle is considered to be very small and negligible.

\section{Local giant permittivity for a single polar molecule}

If we consider the small-scale region on the order of molecular size concerning a nano-hole DNA sequencer, the situation becomes different from that of the above discussion. That is, a DNA molecule consists of four different bases (A: adenine, G: guanine, C: cytosine, T: thymine) attached to a sugar-phosphate backbone, and each individual DNA base has a large dipole moment $P_{B}$ in a small volume $V_{B}$ of the base [4]. By considering a simple well-known 
theory [e.g., see in the Feynman Lectures on Physics [8]] that considers the potential energies $-P_{B} E_{0}$ and $+P_{B} E_{0}$ and the probabilities $C_{B} \mathrm{e}^{P_{B} E_{0} / k T}$ and $C_{B} \mathrm{e}^{-P_{B} E_{0} / k T}$ for the parallel and antiparallel orientations between the dipole and the electric field, respectively, we obtain the expectation value of the moment of the base as $\left\langle P_{B}>=P_{B} C_{B} \mathrm{e}^{P_{B} E_{0} / k T}-P_{B} C_{B} \mathrm{e}^{-P_{B} E_{0} / k T}=\right.$ $P_{B} C_{B}\left(\mathrm{e}^{P_{B} E_{0} / k T}-\mathrm{e}^{-P_{B} E_{0} / k T}\right)=P_{B} \tanh \left(P_{B} E_{0} / k T\right) \simeq P_{B}^{2} E_{0} / k T$, where $C_{B}$ is the proportionality constant that satisfies the conditions that $C_{B} \mathrm{e}^{P_{B} E_{0} / k T}+C_{B} \mathrm{e}^{-P_{B} E_{0} / k T}=1$ (i.e., $\left.C_{B}=\frac{2}{\cosh \frac{P_{B} E_{0}}{k T}}\right)$ and we use the approximation that $\tanh \left(P_{B} E_{0} / k T\right) \simeq P_{B} E_{0} / k T$ at $P_{B} E_{0} / k T \ll 1$. However, the dipole in liquid can generally be oriented in any direction in the applied external electric field. Thus, more precisely, by considering the probability for all the directions [8], we obtain

$$
P_{0}^{B}=\frac{<P_{B}>}{V_{B}}=\frac{P_{B} C_{B}}{V_{B}} \int_{0}^{\pi} n(\theta) \cos \theta 2 \pi \sin \theta d \theta=\frac{P_{B} C_{B} I}{2}
$$

where $\theta$ is the angle between the dipole and the electric field, $n(\theta)=\frac{1}{4 \pi} \mathrm{e}^{\frac{+P_{B} E_{0} \cos \theta}{k T}}$ is the probability per unit solid angle at $\theta, P_{B} E_{0} \cos \theta$ is the potential energy, $I=$ $\int_{-1}^{1} \mathrm{e}^{\frac{+P_{B} E_{0} \cos \theta}{k T}} \cos \theta d(\cos \theta)=\int_{-1}^{1} \mathrm{e}^{a x} x d x=\left.\frac{\mathrm{e}^{a x}}{a}\left(x-\frac{1}{a}\right)\right|_{-1} ^{1}=\frac{2}{a}\left(\cosh a-\frac{1}{a} \sinh a\right), a=\frac{P_{B} E_{0}}{k T}$, and $C_{B}$ satisfies $\frac{C_{B}}{2} \int_{-1}^{1} \mathrm{e}^{a x} d x=\left.\frac{C_{B}}{2 a} \mathrm{e}^{a x}\right|_{-1} ^{1}=\frac{C_{B}}{a} \sinh a=1$ (i.e., $C_{B}=\frac{a}{\sinh a}$ ). Thus, we obtain

$$
P_{0}^{B}=\frac{P_{B}}{V_{B}} \frac{\cosh a-\frac{1}{a} \sinh a}{\sinh a} \simeq \frac{P_{B}^{2} E_{0}}{3 V_{B} k T}
$$

Note that, for the last expression, we use the condition $a \ll 1$; in this case, we can also derive the formula easily by simply considering $\mathrm{e}^{\frac{+P_{B} E_{0} \cos \theta}{k T}} \simeq 1+\frac{P_{B} E_{0} \cos \theta}{k T}$ and $C_{b} \simeq 1$ at $\frac{P_{B} E_{0} \cos \theta}{k T} \ll 1$. Furthermore, from $\epsilon_{0} E_{0}+P_{0}^{B}=\epsilon_{r 2, B} \epsilon_{0} E_{0}$, the relative permittivity of the base is obtained as

$$
\epsilon_{r 2, B} \simeq 1+\frac{P_{B}^{2}}{3 \epsilon_{0} V_{B} k T}
$$

\section{Flow velocity of molecular dipole osmosis based on ICEO}

The formulations of the represented ICEO flow velocities of two-dimensional (2D) and three-dimensional (3D) spherical perfectly polarized particles are identical, as presented by Squires and Bazant [9]. Thus, if we understand the behavior of the ICEO flow of a 2D conductive circular particle, we can understand that of a $3 \mathrm{D}$ conductive spherical particle. 
Similarly, we can understand the behavior of the ICEO flow of a 3D dielectric spherical particle if we understand that of a 2D dielectric circular particle. Thus, by considering Squires and Bazant's formulations [9] with Eq. (1), we can approximate the 2D azimuthal and radial flow velocities around the polarizable particle as follows:

$$
\begin{gathered}
u_{\theta}^{2 D}= \begin{cases}2 \chi U_{0}^{\infty}\left(1-\mathrm{e}^{-\xi}\right) \sin 2 \theta & \left(r_{B} \leq r \leq r_{B}+\lambda_{D}\right), \\
2 \chi U_{0}^{\infty}\left(1-\mathrm{e}^{-1}\right) \frac{\left(r_{B}+\lambda_{D}\right)^{3}}{r^{3}} \sin 2 \theta & \left(r_{B}+\lambda_{D} \leq r\right),\end{cases} \\
u_{r}^{2 D}= \begin{cases}-2 \chi U_{0}^{\infty}\left(1-\mathrm{e}^{-\xi}\right) \cos 2 \theta & \left(r_{B} \leq r \leq r_{B}+\lambda_{D}\right), \\
2 \chi U_{0}^{\infty}\left(1-\mathrm{e}^{-1}\right) \frac{\left(r_{B}+\lambda_{D}\right)\left(\left(r_{B}+\lambda_{D}\right)^{2}-r^{2}\right)}{r^{3}} \cos 2 \theta & \left(r_{B}+\lambda_{D} \leq r\right),\end{cases}
\end{gathered}
$$

where $\xi=\left(r-r_{B}\right) / \lambda$. Note that, in Eqs. (7) and (8), we only consider simple solutions that connect the inside and outside regions as a first approach. Similarly, we can approximate the 3D azimuthal and radial flow velocities around a polarizable particle as follows:

$$
\begin{array}{r}
u_{\theta}^{3 D}= \begin{cases}u_{\theta}^{3 D, \text { inside }}=\frac{9}{8} \chi U_{0}^{\infty}\left(1-\mathrm{e}^{-\xi}\right) \sin 2 \theta & \left(r_{B} \leq r \leq r_{B}+\lambda_{D}\right), \\
\frac{9}{8} \chi U_{0}^{\infty}\left(1-\mathrm{e}^{-1}\right) \frac{\left(r_{B}+\lambda_{D}\right)^{4}}{r^{4}} \sin 2 \theta & \left(r_{B}+\lambda_{D} \leq r\right),\end{cases} \\
u_{r}^{3 D}= \begin{cases}-\frac{9}{16} \chi U_{0}^{\infty}\left(1-\mathrm{e}^{-\xi}\right)(1+3 \cos 2 \theta) & \left(r_{B} \leq r \leq r_{B}+\lambda_{D}\right), \\
\frac{9}{16} \chi U_{0}^{\infty}\left(1-\mathrm{e}^{-1}\right) \frac{\left(r_{B}+\lambda_{D}\right)^{2}\left(\left(r_{B}+\lambda_{D}\right)^{2}-r^{2}\right)}{r^{4}}(1+3 \cos 2 \theta) & \left(r_{B}+\lambda_{D} \leq r\right) .\end{cases}
\end{array}
$$

Therefore, from the above equations, the flow velocities of $2 \mathrm{D}$ and $3 \mathrm{D}$ molecular dipole osmosis based on ICEO can be obtained, and they will be useful for clarifying the physical image of the molecular dipole osmosis.

\section{RESULTS}

\section{A. Flow velocities of molecular dipole osmosis around DNA bases}

Table I shows the predicted flow velocities $U_{0, \epsilon_{r 2}}$ of the molecular dipole osmosis around the DNA bases due to a local giant permittivity $\epsilon_{r 2, B}$ and a small local radius $r_{B}$. Here, $V_{B}$ and $P_{B}$ are the volume and dipole moment, respectively, of each DNA base [4], while $r_{0}^{B}$ is obtained from $r_{0}^{B}=\left(3 V_{B} / 4 \pi\right)^{1 / 3}$. Furthermore, $\epsilon_{r 2, B}$ and $U_{0, \epsilon_{r 2, B}}$ are obtained from Eqs. (6) and (2), respectively, under the condition that $T=300 \mathrm{~K}$ and $\lambda_{D}=10 \mathrm{~nm}$. In Table I, $\epsilon_{r 2, B}$ values of A, G, C, and T are 4.5, 31.9, 40.6, and 16.0, respectively. Namely, 
the local relative permittivities of the DNA bases are much larger than that of the ordinary dielectric material. Consequently, $\epsilon_{r 1, w} / \epsilon_{r 2, B}$ decreases. Furthermore, $r_{B}$ values are 0.317 to $0.342 \mathrm{~nm}$, which are much smaller than $\lambda_{D}(1$ to $100 \mathrm{~nm})$; i.e, $r_{B} / \lambda_{D}$ is usually small for a polar molecule. Thus, the $\chi$ value becomes on the order of unity; thus, we can expect a large ICEO flow around the bases. In fact, the $U_{0, \epsilon_{r 2, B}}$ values at $E_{0}=2 \mathrm{MV} / \mathrm{m}$ are on the order of $1 \mathrm{~mm} / \mathrm{s}$, which are much larger than those at $E_{0}=10 \mathrm{KV} / \mathrm{m}$. Note that the typical electric field for the nanopore problem is approximately $2 \mathrm{MV} / \mathrm{m}$, whereas the typical electric field for standard electrophoresis is approximately $10 \mathrm{kV} / \mathrm{m}$. Note that the required parameters $\left(V_{B}\right.$ and $\left.P_{B}\right)$ are cited from Zwolak and Ventra [4]; i.e., $V_{B}$ denotes the volume of the DNA bases; the $V_{B}$ values are from Table I of Ref. [4]; $P_{B}$ is the theoretically calculated dipole moment of the DNA bases; the $P_{B}$ values are from Table II of Ref. [4].

\begin{tabular}{|c|c|c||c|c|c|c|c|}
\hline Base & $V_{B}\left[\mathrm{~nm}^{3}\right]$ & $P_{B}[\mathrm{D}]$ & $r_{B}[\mathrm{~nm}]$ & $\epsilon_{r 2, B}$ & $\chi^{\lambda_{D}=10 \mathrm{~nm}}$ & $U_{0, \epsilon_{r 2, B}}^{E_{0}=10 \mathrm{KV} / m}[\mathrm{~nm} / \mathrm{s}]$ & $U_{0, \epsilon_{r 2, B}}^{E_{0}=2 \mathrm{M} V / m}[\mathrm{~mm} / \mathrm{s}]$ \\
\hline \hline $\mathrm{A}$ & 0.157 & 2.33 & 0.335 & 4.5 & 0.627 & 14.86 & 0.594 \\
\hline $\mathrm{G}$ & 0.168 & 7.17 & 0.342 & 31.9 & 0.921 & 22.33 & 0.893 \\
\hline $\mathrm{C}$ & 0.133 & 7.22 & 0.317 & 40.6 & 0.941 & 21.11 & 0.845 \\
\hline $\mathrm{T}$ & 0.150 & 4.72 & 0.330 & 16.0 & 0.859 & 20.05 & 0.802 \\
\hline
\end{tabular}

TABLE I. Typical local ICEO flow velocities $U_{0, \epsilon_{r 2}}$ due to a local giant permittivity $\epsilon_{r 2}$ for the bases of a DNA molecule. Here, the required parameters $\left(V_{B}\right.$ and $\left.P_{B}\right)$ are cited from Zwolak and Ventra [4]; i.e., $V_{B}$ denotes the volume of the DNA bases; the $V_{B}$ values are from Table I of Ref. [4]; $P_{B}$ is the theoretically calculated dipole moment of the DNA bases; the $P_{B}$ values are from Table II of Ref. [4]; $r_{0}^{B}$ is the average radius of the base obtained using $r_{0}^{B}=\left(3 V_{B} / 4 \pi\right)^{1 / 3} ; \epsilon_{r 2, B}$ and $U_{0, \epsilon_{r 2, B}}$ are obtained using Eqs. (6) and (2), respectively; we set $T=300 \mathrm{~K}$ and $\lambda_{D}=10 \mathrm{~nm}$ as typical conditions.

\section{B. Characteristics of molecular dipole osmosis based on ICEO}

Figure 2 shows the characteristics of molecular dipole osmosis based on ICEO. Specifically, Figs. 2(a) and 2(b) show the dependences of $\left\langle P_{B}>/ V_{B}\right.$ and $\epsilon_{r, 2}$, respectively, on $E_{0}$ for the DNA bases (A,G,C,T). In Fig. 2(a), the local polarization $\left\langle P_{B}\right\rangle / V_{B}$ increases linearly as $E_{0}$ increases under the condition $\left|E_{0}\right|<10^{7} \mathrm{~V} / \mathrm{m}$. Thus, we can simply approximate 
that $P_{0}^{B} \simeq P_{B}^{2} E_{0} / V_{B} k T$ at $\left|E_{0}\right|<10^{7} \mathrm{~V} / \mathrm{m}$. Consequently, $\epsilon_{r, 2}$ becomes constant at $\left|E_{0}\right|<$ $10^{7} \mathrm{~V} / \mathrm{m}$. Furthermore, Fig. 2(c) shows the dependence of $u_{\theta}^{2 D}$ on $r$ for the DNA bases $(\mathrm{A}, \mathrm{G}, \mathrm{C}, \mathrm{T})$. As shown in Fig. 2(c), the peak flow velocities of $\mathrm{A}$ and $\mathrm{T}$ are significantly different from those of $\mathrm{G}$ and $\mathrm{C}$. Thus, there is a possibility of distinguishing $\mathrm{A}$ and $\mathrm{T}$ from $\mathrm{G}$ and $\mathrm{C}$ using the large difference in the ICEO flow velocities around the bases in a nanopore, although it might be difficult to distinguish $\mathrm{G}$ from $\mathrm{C}$ under the condition that $E_{0}=2 \mathrm{MV} / \mathrm{m}$ and $\lambda_{D}=1 \mathrm{~nm}$. Note that the radii of the bases are almost the same compared with $\lambda_{D}$, and the difference in radius does not work well to distinguish the bases, as pointed out in [4]. Furthermore, Fig. 2(d) shows a predicted 2D flow field around thymine (T) under the condition that $E_{0}=2 \mathrm{MV} / \mathrm{m}$ and $\lambda_{D}=1 \mathrm{~nm}$. In Fig. 2(d), the thick solid line shows the average surface of the thymine molecule, and the thin solid line shows the outside edge of the electric double layer. As shown in Fig. 2(d), we can observe a quadrupolar vortex flow around the thymine molecule in both outside and inside regions, although the flow velocity is assumed to be zero stochastically on the average molecular surface.

\section{Preparative analysis for distinguishing DNA bases}

As mentioned above, each base in a DNA molecule has a unique local permittivity that is different from each other for the typical conditions of the nanopore experiment. However, the local ICEO flow velocity around the base $\left(U_{0}^{B}=U_{0, \epsilon_{r 2}, B}\right)$ depends on not only $E_{0}$ but also $\lambda_{D}$. Thus, it is interesting to clarify the suitable condition for distinguishing the four bases of a DNA molecule through a hole-type sensor using different local ICEO flows around each bases. Figure 3 shows the results of a preparative analysis for distinguishing the DNA bases. Specifically, Figs. 3(a) and 3(b) show the dependences of $U_{o}^{B}$ and $\chi$, respectively, on $\lambda_{D}$ at $E_{0}=2 \mathrm{MV} / \mathrm{m}$, whereas Figs. 3(c) and 3(d) show the dependence of $U_{o}^{B}$ on $E_{0}$ at $\lambda_{D}=1$ and $10 \mathrm{~nm}$. In Fig. 3(a), we find that the condition that $\lambda_{D}=1 \mathrm{~nm}$ at $E_{0}=2 \mathrm{MV} / \mathrm{m}$ is suitable for distinguishing $\mathrm{A}$ and $\mathrm{T}$ from $\mathrm{G}$ and $\mathrm{C}$, as mentioned earlier, although we need to select the condition that $\lambda_{D} \simeq 8 \mathrm{~nm}$ at $E_{0}=2 \mathrm{MV} / \mathrm{m}$ to distinguish the four bases in a DNA molecule in a single measurement. Furthermore, if we want to distinguish only G from $\mathrm{C}$, the condition that $\lambda_{D}=100 \mathrm{~nm}$ at $E_{0}=2 \mathrm{MV} / \mathrm{m}$ is suitable. In addition, since the behavior of the factor $\chi$ is different from that of $U_{0}^{B}$, we need to consider carefully the treatment when we design a DNA sequencer using the ICEO flow difference. Furthermore, 


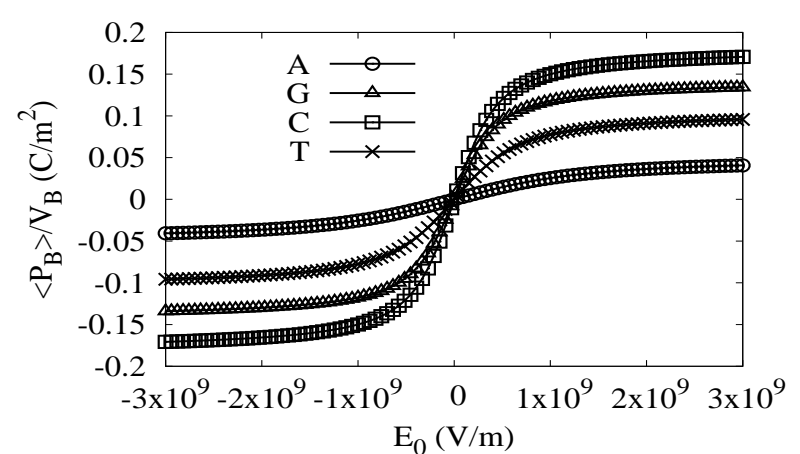

(a) Dependence of $<P_{B}>/ V_{B}$ on $E_{0}$

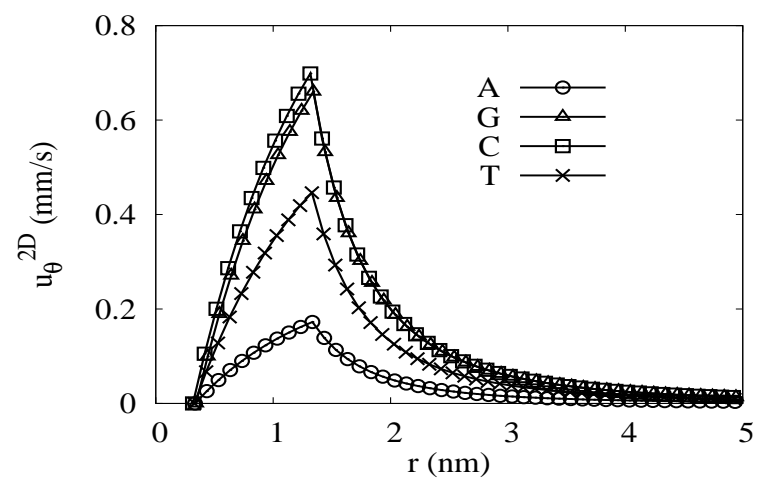

(c) Dependence of $u_{\theta}^{2 D}$ on $r\left(\lambda_{D}=1 \mathrm{~nm}, E_{0}=2\right.$

$$
\mathrm{MV} / \mathrm{m})
$$

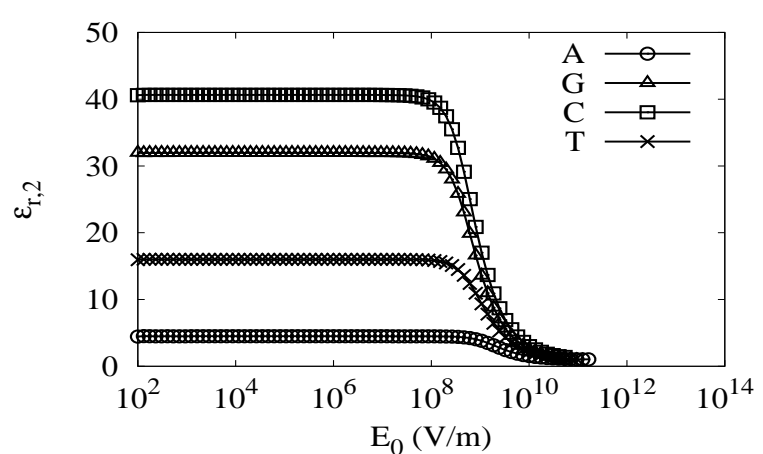

(b) Dependence of $\epsilon_{r, 2}$ on $E_{0}(\lambda=10 \mathrm{~nm})$

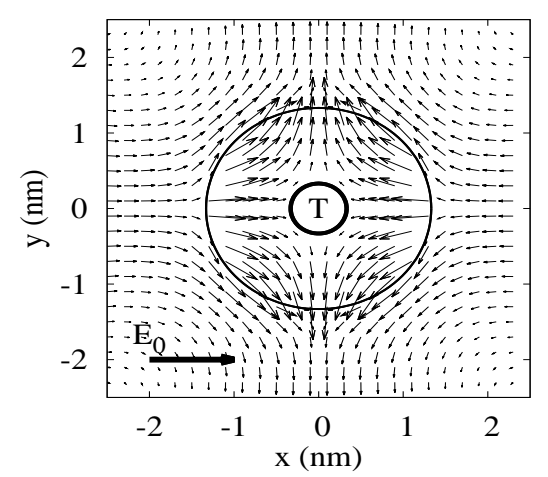

(d) Flow field around "T" $\left(\lambda_{D}=1 \mathrm{~nm}, E_{0}=2\right.$

$\mathrm{MV} / \mathrm{m})$

FIG. 2. Characteristics of molecular dipole osmosis based on ICEO. Here, the values of $<P_{B}>$ $/ V_{B}$ and $\epsilon_{r, 2}$ are calculated using Eqs. (5) and (6), respectively, whereas $u_{\theta}^{2 D}$ is calculated using Eqs. (7) and (8) on the basis of the parameters in Table. I [4].

Figs. 3(c) and 3(d) show the dependences of $U_{o}^{B}$ on $E_{0}$ at $\lambda_{D}=1$ and $8 \mathrm{~nm}$, respectively. From Figs. 3(c) and 3(d), we find that the resolution between $\mathrm{G}$ and $\mathrm{C}$ is not improved even if we increase the intensity of the applied electric field at $\lambda_{D}=1$ and $8 \mathrm{~nm}$. However, Fig. 3(d) preferably shows that the four bases in a DNA molecule can be distinguished using the difference in fluid velocities around bases in a single measurement in a relatively wide range of an electric field of nanopore experiments. 


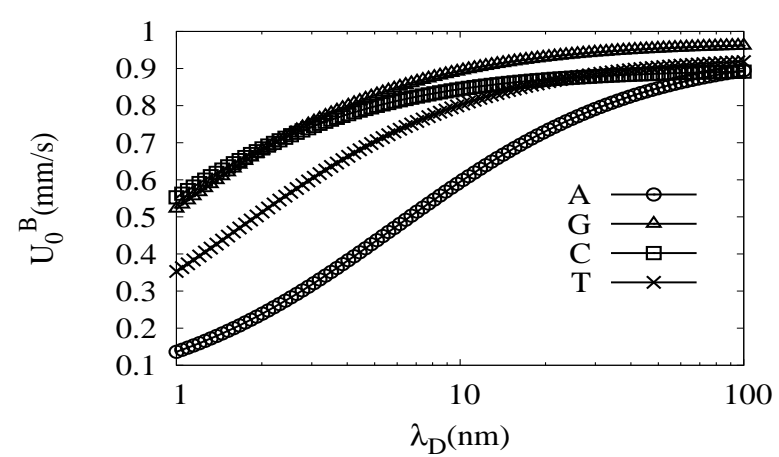

(a) Dependence of $U_{o}^{B}$ on $\lambda_{D}\left(E_{0}=2 \mathrm{MV} / \mathrm{m}\right)$

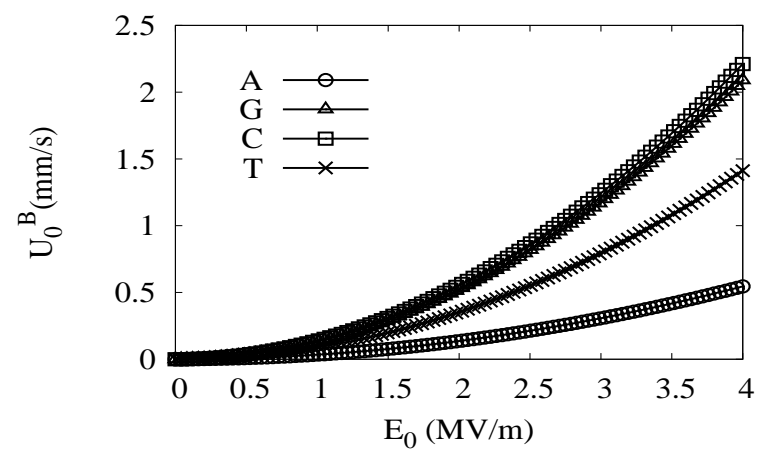

(c) Dependence of $U_{o}^{B}$ on $E_{0}\left(\lambda_{D}=1 \mathrm{~nm}\right)$

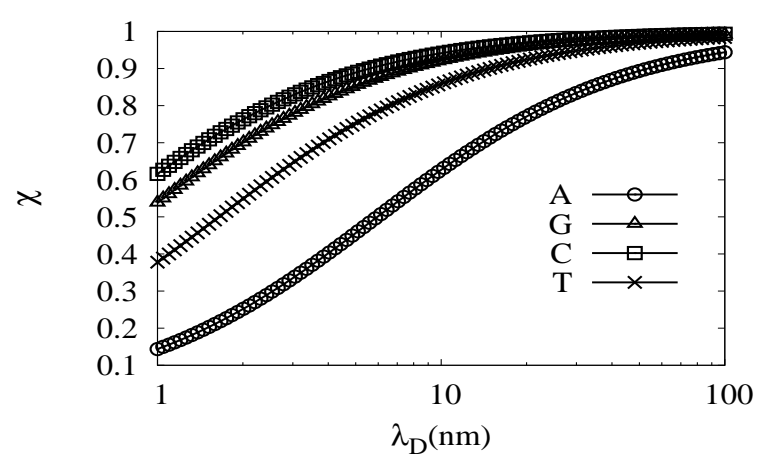

(b) Dependence of $\chi$ on $\lambda_{D}\left(E_{0}=2 \mathrm{MV} / \mathrm{m}\right)$

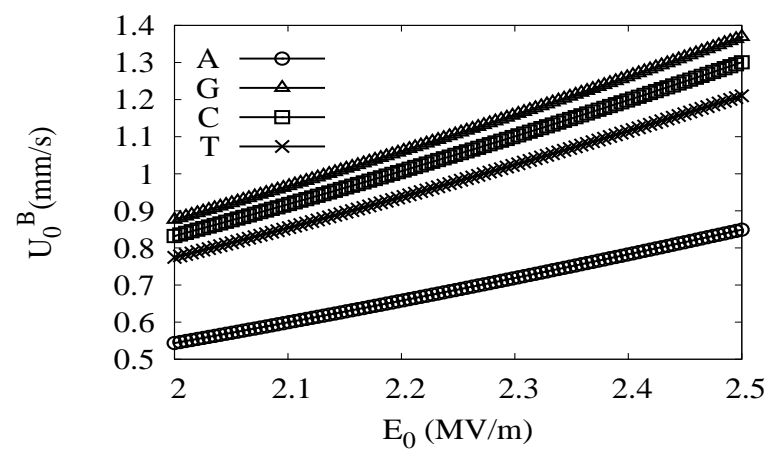

(d) Dependence of $U_{o}^{B}$ on $E_{0}\left(\lambda_{D}=3 \mathrm{~nm}\right)$

FIG. 3. Preparative analysis for distinguishing the DNA bases. Here, $U_{0}^{B}\left(=U_{0, \epsilon_{r 2}, B}\right)$ is calculated [using Eq. (2)] on the basis of the parameters in Table. I.

\section{DISCUSSION}

Although Zhao and Bau [7] already formulated an ICEO flow around a dielectric particle, we have first shown that the ICEO flow velocities around the bases (A, G, C, T) in a DNA molecule can be significantly high in typical nanopore channels because of the local large permittivity of the bases along with the strong electric field. Note that a surprising aspect is that ICEO plays a role at this scale, since the flow scales as size, and is usually negligible for submicron particles, in a typical field; however, the fields in the nanopore problem are enormous (typically $2 \mathrm{MV} / \mathrm{m}$ ), and since ICEO flow scales as $E_{0}^{2}$, the effect could be very large.

The ICEO flow velocity around a dielectric particle [7] is represented by $U_{0, \epsilon_{r 2, B}}=\chi U_{0}^{\infty}=$ $\frac{1}{1+\frac{\epsilon_{r 1, w^{r} B}}{\epsilon_{r 2, B^{\lambda} D}}} \frac{\epsilon_{w} r_{B} E_{0}^{2}}{\mu}$ and $\epsilon_{r 2, B} \simeq 1+\frac{P_{B}^{2} E_{0}}{3 V_{B} k T}$ [i.e., Eqs. (2), (3), (4), and (6)]. Thus, macroscopically, 
the ICEO flow velocity depends on the permittivity of the particle, whereas microscopically it depends on the molecular dipole moment. Therefore, there is a possibility that we can detect the difference in the permittivity (or the molecular dipole moment) of the DNA bases in a nanopore DNA sequencer [4]. In particular, since the ICEO flow around a particle suppresses the Brownian noise in a hole-type sensor [6], the ICEO flow around the DNA bases also effectively suppresses the Brownian noise in a nanopore. Thus, the suppression level of the Brownian noise depends on the ICEO flow velocity around the DNA bases. In other words, our theory suggests that the current signal in a DNA sequencer represents the difference in the surrounding flow velocities that depend on the local dipole moments. Consequently, we have a possibility of distinguishing each bases using the difference in ICEO flows around the bases, and thus the ICEO flow velocity around the bases is probably more important than the real size itself in a nanopore DNA sequencer. However, clarifying the design concept of the nanopore DNA sequencer using ICEO is beyond the scope of this study. We will be clarifying the problem in the future.

The derivation of the dielectric constant of bases relies on statistical mechanics for noninteracting homogeneous macroscopic systems. However, our system is inhomogeneous and of sub-nanometer scale. Thus, these sub-nanometer phenomena might be analyzed using atomistic models. Furthermore, we neglect the dipole moment of water molecules and their interactions. Namely, we need to consider that the dielectric constants of the bases that we obtain are effective ones. Thus, we may need to solve this defect of the theory in the future. However, nowadays, the dielectric constants and flows on the nano scale and atomic scale are generally acceptable and they form part of the theoretical fundamentals for "nanophotonics" and "nanofluidics" [10]. For example, Ghosal [11] successfully analyzed the effect of salt concentration on the electrophoretic speed of a polyelectrolyte through a nanopore of 5.1 $\mathrm{nm}$ radius using the Stokes equation (i.e., macroscopic equation). Furthermore, Okamoto et al. [12] successfully analyzed an X-ray waveguide with a periodic structure of $15 \mathrm{~nm}$ period, by macroscopic simulation based on electromagnetism with the local refractive index of the nanometer region. Thus, we think that our theory is justified.

Furthermore the theory of induced-charge electro-osmosis considers the nonslip boundary condition on the surface of dielectric or conducting particles. However, when we apply this theory to the molecular scale, one might consider that it will be natural to use the slip boundary condition because the surface slip usually enhances electro osmosis. Nevertheless, 
we consider that the nonslip boundary condition is better than the slip boundary condition as long as we rely on a so-called adiabatic approximation that is successfully used in the common argument of the electrophoretic flow resulting from the interaction between the electric field and the charge in the electric double layer for a nanoparticle. Namely, even if the particle is very small (e.g., nanosize) and the Brownian motion cannot be negligible, the nonslip boundary conditions are usually justified in each ensemble that freezes the motion of the particle. Similarly, we consider that the viscous force due to the nonslip boundary conditions plays a crucial role even in the nanoscale region, as pointed out by Ghosal [13].

\section{CONCLUSION}

We have proposed a new mechanism of producing nonlinear electrokinetic vortex flow around a dipole molecule in an electrolyte. In particular, we find that the ICEO flow velocities around the bases (A, G, C, T) that constitute a DNA molecule can be significantly high. because of the local large permittivity due to the large molecular dipole moment in a confined space. We consider that our findings will contribute markedly to the development of innovative next-generation DNA sequencer.

\section{ACKNOWLEDGMENTS}

This work was partially supported by JSPS KAKENHI Grant Number JP16K05650.

[1] M. Z. Bazant and T. M. Squires, Phys. Rev. Lett. 92, 066101 (2004).

[2] T. M. Squires, Lab Chip 9, 2477 (2009).

[3] M. Z. Bazant, M. S. Kilic, B. D. Storey, and A. Ajdari, Adv. Colloid Interface Sci. 152, 48 (2009).

[4] M. Zwolak and M. D. Ventra, Rev. Mod. Phys. 80, 141 (2008).

[5] H. Sugioka, Colloids and Surfaces A: Physicochem. Eng. Aspects 27, 440 (2014).

[6] H. Sugioka, Physics of Fluids 28, 032003 (2016), http://dx.doi.org/10.1063/1.4943495.

[7] H. Zhao and H. Bau, Langmuir 23, 4053 (2007). 
[8] R. P. Feynman, R. B. Leighton, and M. L. Sands, The Feynman Lectures on Physics (New Millennium Edition, http://www.feynmanlectures.info/, 1965).

[9] T. M. Squires and M. Z. Bazant, J. Fluid Mech. 509, 217 (2004).

[10] J. C. T. Eijkel and A. v. d. Berg, Microfluidics and Nanofluidics 1, 249 (2005).

[11] S. Ghosal, Phys. Rev. Lett. 98, 238104 (2007).

[12] K. Okamoto, T. Noma, A. Komoto, W. Kubo, M. Takahashi, A. Iida, and H. Miyata, Phys. Rev. Lett. 109, 233907 (2012).

[13] S. Ghosal, Phys. Rev. E 76, 061916 (2007). 